Acta Crystallographica Section E

Structure Reports

Online

ISSN 1600-5368

\section{4'-tert-Butyl-5-chloro-3H-spiro[1,3- benzothiazole-2,1'-cyclohexane]}

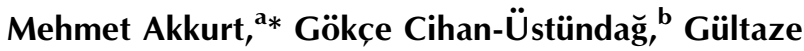 \\ Capan, ${ }^{\text {b }}$ Yılmaz Dağdemir ${ }^{\mathrm{a}}$ and Muhammad Nawaz Tahir ${ }^{\mathrm{c}}$

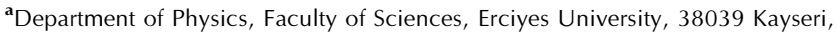 \\ Turkey, ${ }^{\mathbf{b}}$ Department of Pharmaceutical Chemistry, Faculty of Pharmacy, Istanbul \\ University, 34116 Beyazıt, Istanbul, Turkey, and ${ }^{\mathbf{c}}$ Department of Physics, University \\ of Sargodha, Sargodha, Pakistan \\ Correspondence e-mail: akkurt@erciyes.edu.tr
}

Received 14 April 2012; accepted 19 April 2012

Key indicators: single-crystal X-ray study; $T=296 \mathrm{~K}$; mean $\sigma(\mathrm{C}-\mathrm{C})=0.003 \AA$; $R$ factor $=0.052 ; w R$ factor $=0.128 ;$ data-to-parameter ratio $=21.5$.

In the title compound, $\mathrm{C}_{16} \mathrm{H}_{22} \mathrm{ClNS}$, the nine-membered 2,3dihydro-1,3-benzothiazole ring system is essentially planar, with a maximum deviation of 0.025 (2) $\AA$ for the $\mathrm{N}$ atom. Its plane is almost perpendicular to the main plane of the substituted cyclohexane ring, which adopts a chair conformation. In the crystal, the molecules are linked by $\mathrm{C}-\mathrm{H} \cdots \pi$ interactions.

\section{Related literature}

For the pharmacological activity of benzothiazole derivatives, see: Coudert et al. (1988); Karalı et al. (2010); Palmer et al. (1971). For the crystal structures of similar compounds, see, for example: Akkurt et al. (2010); Aryai et al. (1976); Karalı et al. (2010). For standard values of bond lengths, see: Allen et al. (1987). For details of ring-puckering analysis, see: Cremer \& Pople (1975).

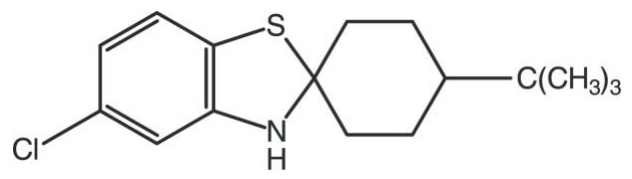

\section{Experimental}

Crystal data

$\mathrm{C}_{16} \mathrm{H}_{22} \mathrm{ClNS}$

$M_{r}=295.87$

Monoclinic, $P 2_{1} / c$

$a=15.2810(18) \AA$

$b=8.9830(8) \AA$

$c=11.8750(13) \AA$

$\beta=109.580$ (3)
Data collection

Bruker Kappa APEXII CCD diffractometer

Absorption correction: multi-scan (SADABS; Bruker, 2005)

$T_{\min }=0.915, T_{\max }=0.935$

Refinement

$R\left[F^{2}>2 \sigma\left(F^{2}\right)\right]=0.052$

$w R\left(F^{2}\right)=0.128$

$S=1.02$

3849 reflections

179 parameters

1 restraint

14074 measured reflections 3849 independent reflections 2330 reflections with $I>2 \sigma(I)$ $R_{\text {int }}=0.043$

\section{Table 1}

Hydrogen-bond geometry $\left(\AA{ }^{\circ}\right)$.

$\mathrm{Cg} 1$ is the centroid of the $\mathrm{C} 1-\mathrm{C} 6$ benzene ring.

\begin{tabular}{lllll}
\hline$D-\mathrm{H} \cdots A$ & $D-\mathrm{H}$ & $\mathrm{H} \cdots A$ & $D \cdots A$ & $D-\mathrm{H} \cdots A$ \\
\hline $\mathrm{C} 8-\mathrm{H} 8 B \cdots C g 1^{\mathrm{i}}$ & 0.97 & 2.84 & $3.796(2)$ & 169 \\
\hline
\end{tabular}

Symmetry code: (i) $-x, y+\frac{1}{2},-z+\frac{1}{2}$.

Data collection: APEX2 (Bruker, 2009); cell refinement: SAINT (Bruker, 2009); data reduction: $S A I N T$; program(s) used to solve structure: SHELXS97 (Sheldrick, 2008); program(s) used to refine structure: SHELXL97 (Sheldrick, 2008); molecular graphics: ORTEP-3 for Windows (Farrugia, 1997) and PLATON (Spek, 2009); software used to prepare material for publication: Win $G X$ (Farrugia, 1999) and PLATON.

The authors acknowledge the provision of funds for the purchase of a diffractometer and encouragement by Dr Muhammad Akram Chaudhary, Vice Chancellor, University of Sargodha, Pakistan.

Supplementary data and figures for this paper are available from the IUCr electronic archives (Reference: SU2411).

\section{References}

Akkurt, M., Karaca, S., Ermut, G., Karalı, N. \& Büyükgüngör, O. (2010). Acta Cryst. E66, o399-0400.

Allen, F. H., Kennard, O., Watson, D. G., Brammer, L., Orpen, A. G. \& Taylor, R. (1987). J. Chem. Soc. Perkin Trans. 2, pp. S1-19.

Aryai, V. P., Nair, M. G., Wasaiwalla, Y. H. \& Shenoy, S. J. (1976). Indian J. Chem. Sect. B, 14, 984-987.

Bruker (2005). SADABS. Bruker AXS Inc., Madison, Wisconsin, USA.

Bruker (2009). APEX2 and SAINT. Bruker AXS Inc., Madison, Wisconsin, USA.

Coudert, P., Couquelet, J., Sudre, O. \& Bastide, J. (1988). J. Pharm. Belg. 43 258-262.

Cremer, D. \& Pople, J. A. (1975). J. Am. Chem. Soc. 97, 1354-1358.

Farrugia, L. J. (1997). J. Appl. Cryst. 30, 565.

Farrugia, L. J. (1999). J. Appl. Cryst. 32, 837-838.

Karalı, N., Güzel, Ö., Özsoy, N., Özbey, S. \& Salman, A. (2010). Eur. J. Med. Chem. 45, 1068-1077.

Palmer, P. J., Trigg, R. B. \& Warrington, J. V. (1971). J. Med. Chem. 14, 248-251.

Sheldrick, G. M. (2008). Acta Cryst. A64, 112-122.

Spek, A. L. (2009). Acta Cryst. D65, 148-155. 


\section{supporting information}

Acta Cryst. (2012). E68, o1542 [doi:10.1107/S1600536812017539]

\section{4'-tert-Butyl-5-chloro-3H-spiro[1,3-benzothiazole-2,1'-cyclohexane] \\ Mehmet Akkurt, Gökçe Cihan-Üstündağ, Gültaze Çapan, Yılmaz Dağdemir and Muhammad Nawaz Tahir}

\section{S1. Comment}

The condensation of aldehydes and ketones with 2-aminothiophenoles lead to benzothiazolines and spirobenzothiazolines which are reported to exhibit antitubercular (Palmer et al., 1971), analgesic (Coudert et al., 1988) and antioxidant (Karalı et al., 2010) properties. The reactivity of cyclic ketones towards 2-aminothiophenoles has also been examined and the structure of the end products has been discussed (Aryai et al., 1976; Coudert et al., 1988; Akkurt et al., 2010; Karalı et al., 2010). Prompted by the above observations, we report here the synthesis, spectroscopic and crystal structure of the title compound.

As shown in Fig. 1, the $\mathrm{C} 7-\mathrm{C} 12$ cyclohexane ring of the title compound adopts a chair conformation [puckering parameters (Cremer \& Pople, 1975): $\mathrm{Q}_{\mathrm{T}}=0.564$ (2) $\AA, \theta=176.5(2)^{\circ}$ and $\left.\varphi=4(4)^{\circ}\right]$. The mean plane of the 2,3-dihydro-1,3-benzothiazole ring system [max. deviation: -0.025 (2) $\AA$ for N1] is almost perpendicular with a dihedral angle of 89.39 (5) ${ }^{\circ}$ to the main plane formed by the $\mathrm{C} 8, \mathrm{C} 9, \mathrm{C} 11$ and $\mathrm{C} 12$ atoms of the cyclohexane ring. The bond lengths (Allen et al., 1987) and bond angles are within the expected values.

The crystal packing is stabilized by $\mathrm{C}-\mathrm{H} \cdots \pi$ interactions (Table 1 and Fig. 2).

\section{S2. Experimental}

A mixture of 2-amino-4-chlorothiophenol $(0.01 \mathrm{~mol})$ and 4-tert-butylcyclohexanone $(0.01 \mathrm{~mol})$ in absolute ethanol $(50$ $\mathrm{ml}$ ) was refluxed on a water bath for $8 \mathrm{~h}$. The solvent was evaporated in a crystallizing dish at room temperature and the residue was recrystallized twice from ethanol, giving X-ray quality crystals [Yield: 24.3\%, m.p.: 453-455 K]. Analysis calculated for $\mathrm{C}_{16} \mathrm{H}_{22} \mathrm{ClNS}$ : C 64.95, H 7.49, N 4.73\%. Found: C 64.91, H 7.47, N 4.64\%. Spectroscopic data for the title compound are given in the archive CIF.

\section{S3. Refinement}

The NH H atom was located in a difference Fourier map and freely refined. C-bound $\mathrm{H}$ atoms were placed in calculated positions and treated as riding atoms : $\mathrm{C}-\mathrm{H}=0.93,0.96,0.97$ and $0.98 \AA$, for the aromatic, methyl, methylene and methine $\mathrm{H}$ atoms, respectively, with $U_{\mathrm{iso}}(\mathrm{H})=x U_{\mathrm{eq}}(\mathrm{C}), x=1.5$ for methyl $\mathrm{H}$ atoms and $=1.2$ for other $\mathrm{H}$ atoms. 


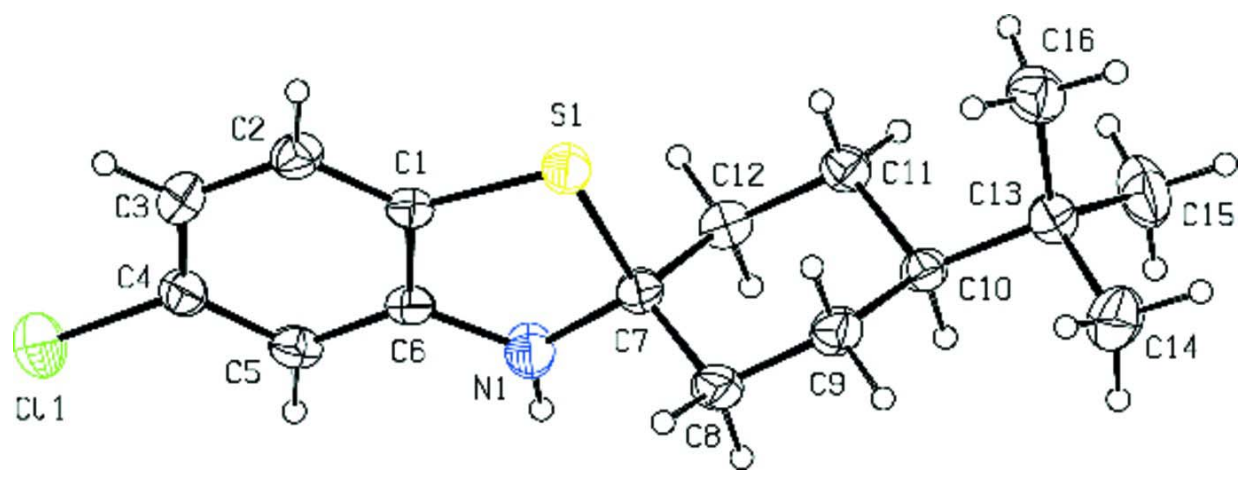

Figure 1

The molecular structure of the title molecule, with the atom numbering. Displacement ellipsoids are drawn at the $30 \%$ probability level.

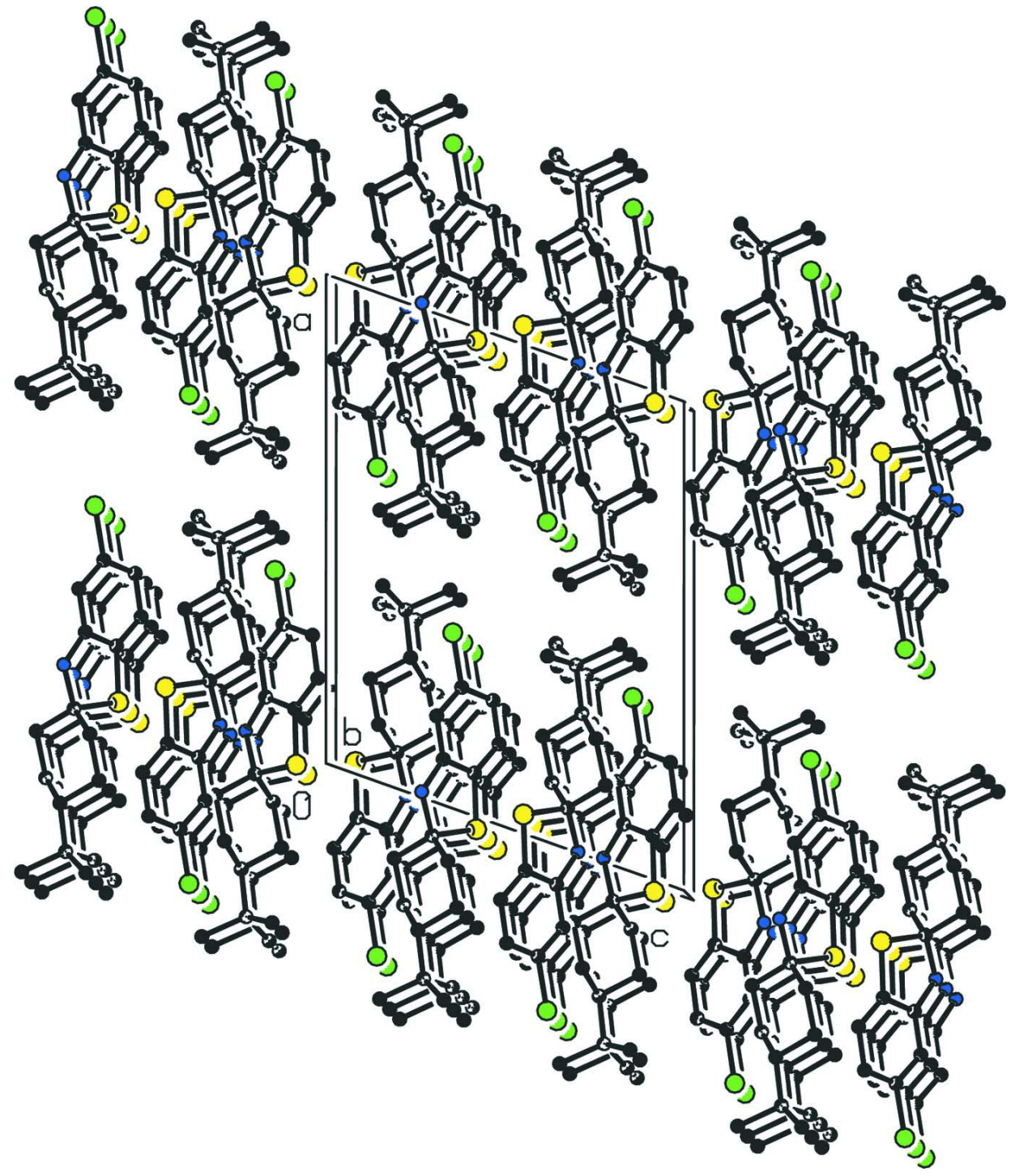




\section{Figure 2}

The crystal packing of the title compound viewing along $b$ axis [H atoms have been omitted for clarity].

\section{4'-tert-Butyl-5-chloro-3H-spiro[1,3-benzothiazole-2,1'- cyclohexane]}

Crystal data

$\mathrm{C}_{16} \mathrm{H}_{22} \mathrm{ClNS}$

$M_{r}=295.87$

Monoclinic, $P 2_{1} / c$

Hall symbol: $-\mathrm{P} 2 \mathrm{ybc}$

$a=15.2810(18) \AA$

$b=8.9830(8) \AA$

$c=11.8750(13) \AA$

$\beta=109.580(3)^{\circ}$

$V=1535.8(3) \AA^{3}$

$Z=4$

Data collection

Bruker Kappa APEXII CCD diffractometer

Radiation source: fine-focus sealed tube

Graphite monochromator

$\omega$ scans

Absorption correction: multi-scan

(SADABS; Bruker, 2005)

$T_{\min }=0.915, T_{\max }=0.935$

Refinement

Refinement on $F^{2}$

Least-squares matrix: full

$R\left[F^{2}>2 \sigma\left(F^{2}\right)\right]=0.052$

$w R\left(F^{2}\right)=0.128$

$S=1.02$

3849 reflections

179 parameters

1 restraint

Primary atom site location: structure-invariant

direct methods
$F(000)=632$

$D_{\mathrm{x}}=1.280 \mathrm{Mg} \mathrm{m}^{-3}$

Mo $K \alpha$ radiation, $\lambda=0.71073 \AA$

Cell parameters from 776 reflections

$\theta=3.3-19.5^{\circ}$

$\mu=0.37 \mathrm{~mm}^{-1}$

$T=296 \mathrm{~K}$

Prism, colourless

$0.27 \times 0.20 \times 0.18 \mathrm{~mm}$

14074 measured reflections

3849 independent reflections

2330 reflections with $I>2 \sigma(I)$

$R_{\text {int }}=0.043$

$\theta_{\text {max }}=28.5^{\circ}, \theta_{\min }=2.7^{\circ}$

$h=-20 \rightarrow 20$

$k=-11 \rightarrow 10$

$l=-15 \rightarrow 15$

Secondary atom site location: difference Fourier map

Hydrogen site location: inferred from neighbouring sites

$\mathrm{H}$ atoms treated by a mixture of independent and constrained refinement

$w=1 /\left[\sigma^{2}\left(F_{\mathrm{o}}^{2}\right)+(0.0473 P)^{2}+0.2285 P\right]$

where $P=\left(F_{\mathrm{o}}^{2}+2 F_{\mathrm{c}}^{2}\right) / 3$

$(\Delta / \sigma)_{\max }<0.001$

$\Delta \rho_{\max }=0.25 \mathrm{e} \AA^{-3}$

$\Delta \rho_{\min }=-0.24$ e $\AA^{-3}$

\section{Special details}

Experimental. Spectroscopic data for the title compound: IR $(\mathrm{KBr}) v=3370(\mathrm{~N}-\mathrm{H}), 2962,2912,2862(\mathrm{C}-\mathrm{H}), 1585$, 1571, 1473, $1442(\mathrm{C}=\mathrm{C}) \mathrm{cm}^{-1}$; $1 \mathrm{H}$-NMR (DMSO-d6, $\left.500 \mathrm{MHz}\right) \mathrm{d}=0.83-0.86$ (9H, m, 4'-C(CH3)3-cyc.), 0.95-1.02 (1H, $\mathrm{m}, \mathrm{CH} / \mathrm{CH} 2$-сус.), $1.09-1.36$ ( $2 \mathrm{H}, \mathrm{m}, \mathrm{CH} / \mathrm{CH} 2$-сyc.), 1.58-1.72 (4H, m, CH/CH2-cyc.), 2.15-2.22 (2H, m, CH/CH2cyc.), 6.40, 6.47 (1H, 2 d, J=2.0 Hz, H4-bt.), 6.50 (1H, dd, J=8.1, 2.0 Hz, H6-bt.), 6.90 (1H, d, J=7.8 Hz, H7-bt.), 6.73, $6.97(1 \mathrm{H}, 2 \mathrm{~s}, \mathrm{NH})$ p.p.m. (cyc.=cyclohexane, bt.=benzothiazole).

Geometry. Bond distances, angles etc. have been calculated using the rounded fractional coordinates. All su's are estimated from the variances of the (full) variance-covariance matrix. The cell e.s.d.'s are taken into account in the estimation of distances, angles and torsion angles

Refinement. Refinement on $F^{2}$ for ALL reflections except those flagged by the user for potential systematic errors. Weighted $R$-factors $w R$ and all goodnesses of fit $S$ are based on $F^{2}$, conventional $R$-factors $R$ are based on $F$, with $F$ set to zero for negative $F^{2}$. The observed criterion of $F^{2}>\sigma\left(F^{2}\right)$ is used only for calculating $-R$-factor-obs etc. and is not relevant to the choice of reflections for refinement. $R$-factors based on $F^{2}$ are statistically about twice as large as those based on $F$, and $R$-factors based on ALL data will be even larger. 
Fractional atomic coordinates and isotropic or equivalent isotropic displacement parameters $\left(\AA^{2}\right)$

\begin{tabular}{|c|c|c|c|c|}
\hline & $x$ & $y$ & $z$ & $U_{\text {iso }} * / U_{\text {eq }}$ \\
\hline $\mathrm{Cl1}$ & $-0.35486(5)$ & $0.80459(8)$ & $0.13722(7)$ & $0.0761(3)$ \\
\hline S1 & $0.02827(4)$ & $0.69814(6)$ & $0.07426(5)$ & $0.0503(2)$ \\
\hline N1 & $-0.00626(14)$ & $0.8895(2)$ & $0.2213(2)$ & $0.0628(8)$ \\
\hline $\mathrm{C} 1$ & $-0.08493(14)$ & $0.7159(2)$ & $0.07911(17)$ & $0.0369(7)$ \\
\hline $\mathrm{C} 2$ & $-0.16334(16)$ & $0.6409(2)$ & $0.0125(2)$ & $0.0474(8)$ \\
\hline $\mathrm{C} 3$ & $-0.24750(16)$ & $0.6684(2)$ & $0.0286(2)$ & $0.0515(8)$ \\
\hline $\mathrm{C} 4$ & $-0.25028(16)$ & $0.7710(2)$ & $0.1132(2)$ & $0.0469(8)$ \\
\hline $\mathrm{C} 5$ & $-0.17272(16)$ & $0.8482(2)$ & $0.1807(2)$ & $0.0447(7)$ \\
\hline C6 & $-0.08920(15)$ & $0.8210(2)$ & $0.16337(18)$ & $0.0390(7)$ \\
\hline $\mathrm{C} 7$ & $0.07535(15)$ & $0.8422(2)$ & $0.1936(2)$ & $0.0442(7)$ \\
\hline $\mathrm{C} 8$ & $0.11774(16)$ & $0.9705(2)$ & $0.1463(2)$ & $0.0519(8)$ \\
\hline $\mathrm{C} 9$ & $0.20566(15)$ & $0.9285(2)$ & $0.1218(2)$ & $0.0500(8)$ \\
\hline $\mathrm{C} 10$ & $0.27931(14)$ & $0.8647(2)$ & $0.23261(18)$ & $0.0408(7)$ \\
\hline C11 & $0.23620(15)$ & $0.7316(2)$ & 0.27578 (19) & $0.0438(7)$ \\
\hline $\mathrm{C} 12$ & $0.14820(15)$ & $0.7730(2)$ & $0.30136(19)$ & $0.0467(8)$ \\
\hline C13 & $0.37389(16)$ & $0.8291(2)$ & $0.2176(2)$ & $0.0509(8)$ \\
\hline C14 & $0.4048(2)$ & $0.9596(3)$ & $0.1572(3)$ & $0.0845(14)$ \\
\hline $\mathrm{C} 15$ & $0.44715(18)$ & $0.8047(3)$ & $0.3408(2)$ & $0.0800(11)$ \\
\hline $\mathrm{C} 16$ & $0.36933(18)$ & $0.6896(3)$ & $0.1415(2)$ & $0.0651(10)$ \\
\hline $\mathrm{H} 1 \mathrm{~N}$ & $-0.0005(17)$ & $0.955(2)$ & $0.2742(16)$ & $0.070(8)^{*}$ \\
\hline $\mathrm{H} 2$ & -0.16000 & 0.57120 & -0.04380 & $0.0570^{*}$ \\
\hline H3 & -0.30100 & 0.61850 & -0.01690 & $0.0620 *$ \\
\hline H5 & -0.17650 & 0.91750 & 0.23700 & $0.0540 *$ \\
\hline H8A & 0.07240 & 1.00700 & 0.07300 & $0.0620 *$ \\
\hline H8B & 0.13160 & 1.05100 & 0.20410 & $0.0620 *$ \\
\hline H9A & 0.23040 & 1.01600 & 0.09520 & $0.0600 *$ \\
\hline H9B & 0.19090 & 0.85550 & 0.05810 & $0.0600 *$ \\
\hline $\mathrm{H} 10$ & 0.29130 & 0.94100 & 0.29500 & $0.0490 *$ \\
\hline H11A & 0.22180 & 0.65430 & 0.21530 & $0.0520^{*}$ \\
\hline H11B & 0.28120 & 0.69160 & 0.34790 & $0.0520 *$ \\
\hline $\mathrm{H} 12 \mathrm{~A}$ & 0.12260 & 0.68450 & 0.32520 & $0.0560^{*}$ \\
\hline H12B & 0.16360 & 0.84290 & 0.36740 & $0.0560^{*}$ \\
\hline H14A & 0.46750 & 0.94360 & 0.15950 & $0.1270^{*}$ \\
\hline H14B & 0.40160 & 1.05020 & 0.19850 & $0.1270 *$ \\
\hline $\mathrm{H} 14 \mathrm{C}$ & 0.36470 & 0.96690 & 0.07550 & $0.1270 *$ \\
\hline $\mathrm{H} 15 \mathrm{~A}$ & 0.50660 & 0.78790 & 0.33200 & $0.1200^{*}$ \\
\hline H15B & 0.43040 & 0.71970 & 0.37820 & $0.1200^{*}$ \\
\hline $\mathrm{H} 15 \mathrm{C}$ & 0.45040 & 0.89120 & 0.38960 & $0.1200^{*}$ \\
\hline H16A & 0.31930 & 0.69930 & 0.06700 & $0.0980 *$ \\
\hline H16B & 0.35900 & 0.60370 & 0.18350 & $0.0980 *$ \\
\hline $\mathrm{H} 16 \mathrm{C}$ & 0.42680 & 0.67840 & 0.12620 & $0.0980 *$ \\
\hline
\end{tabular}


Atomic displacement parameters $\left(\AA^{2}\right)$

\begin{tabular}{lllllll}
\hline & $U^{11}$ & $U^{22}$ & $U^{33}$ & $U^{12}$ & $U^{13}$ & $U^{23}$ \\
\hline C11 & $0.0543(4)$ & $0.0816(5)$ & $0.1046(6)$ & $0.0027(3)$ & $0.0428(4)$ & $-0.0018(4)$ \\
S1 & $0.0456(3)$ & $0.0510(4)$ & $0.0568(4)$ & $-0.0017(3)$ & $0.0203(3)$ & $-0.0182(3)$ \\
N1 & $0.0467(12)$ & $0.0636(14)$ & $0.0770(15)$ & $-0.0030(10)$ & $0.0191(11)$ & $-0.0390(12)$ \\
C1 & $0.0441(12)$ & $0.0319(11)$ & $0.0363(11)$ & $0.0025(9)$ & $0.0158(9)$ & $0.0017(8)$ \\
C2 & $0.0533(14)$ & $0.0404(12)$ & $0.0483(13)$ & $-0.0035(10)$ & $0.0168(11)$ & $-0.0086(10)$ \\
C3 & $0.0460(14)$ & $0.0488(14)$ & $0.0571(15)$ & $-0.0096(11)$ & $0.0139(12)$ & $-0.0054(11)$ \\
C4 & $0.0438(13)$ & $0.0414(12)$ & $0.0586(15)$ & $0.0046(10)$ & $0.0214(11)$ & $0.0072(10)$ \\
C5 & $0.0531(14)$ & $0.0361(11)$ & $0.0492(13)$ & $0.0061(10)$ & $0.0229(11)$ & $-0.0011(9)$ \\
C6 & $0.0455(13)$ & $0.0295(10)$ & $0.0412(12)$ & $0.0022(9)$ & $0.0133(10)$ & $-0.0016(9)$ \\
C7 & $0.0402(13)$ & $0.0395(12)$ & $0.0517(13)$ & $-0.0018(9)$ & $0.0139(11)$ & $-0.0117(10)$ \\
C8 & $0.0483(14)$ & $0.0382(12)$ & $0.0580(15)$ & $0.0040(10)$ & $0.0030(11)$ & $0.0069(10)$ \\
C9 & $0.0518(14)$ & $0.0434(12)$ & $0.0515(14)$ & $-0.0031(10)$ & $0.0131(11)$ & $0.0118(10)$ \\
C10 & $0.0424(12)$ & $0.0352(11)$ & $0.0411(12)$ & $-0.0016(9)$ & $0.0091(10)$ & $-0.0016(9)$ \\
C11 & $0.0465(13)$ & $0.0404(12)$ & $0.0400(12)$ & $0.0039(10)$ & $0.0087(10)$ & $0.0094(9)$ \\
C12 & $0.0554(15)$ & $0.0414(12)$ & $0.0440(13)$ & $-0.0032(10)$ & $0.0176(11)$ & $-0.0010(9)$ \\
C13 & $0.0451(14)$ & $0.0488(13)$ & $0.0572(15)$ & $0.0010(10)$ & $0.0151(11)$ & $-0.0043(11)$ \\
C14 & $0.070(2)$ & $0.0732(19)$ & $0.126(3)$ & $-0.0153(15)$ & $0.0536(19)$ & $0.0001(18)$ \\
C15 & $0.0468(16)$ & $0.105(2)$ & $0.077(2)$ & $0.0111(15)$ & $0.0061(15)$ & $-0.0186(16)$ \\
C16 & $0.0638(17)$ & $0.0669(16)$ & $0.0687(17)$ & $0.0053(13)$ & $0.0278(14)$ & $-0.0127(13)$ \\
& & & & & &
\end{tabular}

Geometric parameters $\left(A,{ }^{\circ}\right)$

\begin{tabular}{llll}
\hline $\mathrm{C} 11-\mathrm{C} 4$ & $1.741(3)$ & $\mathrm{C} 2-\mathrm{H} 2$ & 0.9300 \\
$\mathrm{~S} 1-\mathrm{C} 1$ & $1.757(2)$ & $\mathrm{C} 3-\mathrm{H} 3$ & 0.9300 \\
$\mathrm{~S} 1-\mathrm{C} 7$ & $1.875(2)$ & $\mathrm{C} 5-\mathrm{H} 5$ & 0.9300 \\
$\mathrm{~N} 1-\mathrm{C} 6$ & $1.369(3)$ & $\mathrm{C} 8-\mathrm{H} 8 \mathrm{~A}$ & 0.9700 \\
$\mathrm{~N} 1-\mathrm{C} 7$ & $1.456(3)$ & $\mathrm{C} 8-\mathrm{H} 8 \mathrm{~B}$ & 0.9700 \\
$\mathrm{~N} 1-\mathrm{H} 1 \mathrm{~N}$ & $0.844(18)$ & $\mathrm{C} 9-\mathrm{H} 9 \mathrm{~A}$ & 0.9700 \\
$\mathrm{C} 1-\mathrm{C} 2$ & $1.371(3)$ & $\mathrm{C} 9-\mathrm{H} 9 \mathrm{~B}$ & 0.9700 \\
$\mathrm{C} 1-\mathrm{C} 6$ & $1.393(3)$ & $\mathrm{C} 10-\mathrm{H} 10$ & 0.9800 \\
$\mathrm{C} 2-\mathrm{C} 3$ & $1.384(4)$ & $\mathrm{C} 11-\mathrm{H} 11 \mathrm{~A}$ & 0.9700 \\
$\mathrm{C} 3-\mathrm{C} 4$ & $1.375(3)$ & $\mathrm{C} 11-\mathrm{H} 11 \mathrm{~B}$ & 0.9700 \\
$\mathrm{C} 4-\mathrm{C} 5$ & $1.375(3)$ & $\mathrm{C} 12-\mathrm{H} 12 \mathrm{~A}$ & 0.9700 \\
$\mathrm{C} 5-\mathrm{C} 6$ & $1.381(3)$ & $\mathrm{C} 12-\mathrm{H} 12 \mathrm{~B}$ & 0.9700 \\
$\mathrm{C} 7-\mathrm{C} 12$ & $1.519(3)$ & $\mathrm{C} 14-\mathrm{H} 14 \mathrm{~A}$ & 0.9600 \\
$\mathrm{C} 7-\mathrm{C} 8$ & $1.519(3)$ & $\mathrm{C} 14-\mathrm{H} 14 \mathrm{~B}$ & 0.9600 \\
$\mathrm{C} 8-\mathrm{C} 9$ & $1.515(3)$ & $\mathrm{C} 14-\mathrm{H} 14 \mathrm{C}$ & 0.9600 \\
$\mathrm{C} 9-\mathrm{C} 10$ & $1.528(3)$ & $\mathrm{C} 15-\mathrm{H} 15 \mathrm{~A}$ & 0.9600 \\
$\mathrm{C} 10-\mathrm{C} 13$ & $1.548(3)$ & $\mathrm{C} 15-\mathrm{H} 15 \mathrm{~B}$ & 0.9600 \\
$\mathrm{C} 10-\mathrm{C} 11$ & $\mathrm{C} 15-\mathrm{H} 15 \mathrm{C}$ & 0.9600 \\
$\mathrm{C} 11-\mathrm{C} 12$ & $1.534(3)$ & $\mathrm{C} 16-\mathrm{H} 16 \mathrm{~A}$ & 0.9600 \\
$\mathrm{C} 13-\mathrm{C} 15$ & $1.521(3)$ & $\mathrm{C} 16-\mathrm{H} 16 \mathrm{~B}$ & 0.9600 \\
$\mathrm{C} 13-\mathrm{C} 16$ & $1.531(3)$ & $\mathrm{C} 16-\mathrm{H} 16 \mathrm{C}$ & 0.9600 \\
$\mathrm{C} 13-\mathrm{C} 14$ & $1.533(3)$ & &
\end{tabular}




\begin{tabular}{|c|c|c|c|}
\hline $\mathrm{C} 1-\mathrm{S} 1-\mathrm{C} 7$ & $92.59(10)$ & $\mathrm{C} 7-\mathrm{C} 8-\mathrm{H} 8 \mathrm{~A}$ & 109.00 \\
\hline $\mathrm{C} 6-\mathrm{N} 1-\mathrm{C} 7$ & $118.31(19)$ & $\mathrm{C} 7-\mathrm{C} 8-\mathrm{H} 8 \mathrm{~B}$ & 109.00 \\
\hline $\mathrm{C} 6-\mathrm{N} 1-\mathrm{H} 1 \mathrm{~N}$ & $122.4(18)$ & $\mathrm{C} 9-\mathrm{C} 8-\mathrm{H} 8 \mathrm{~A}$ & 109.00 \\
\hline $\mathrm{C} 7-\mathrm{N} 1-\mathrm{H} 1 \mathrm{~N}$ & $119.2(18)$ & $\mathrm{C} 9-\mathrm{C} 8-\mathrm{H} 8 \mathrm{~B}$ & 109.00 \\
\hline $\mathrm{C} 2-\mathrm{C} 1-\mathrm{C} 6$ & $120.3(2)$ & $\mathrm{H} 8 \mathrm{~A}-\mathrm{C} 8-\mathrm{H} 8 \mathrm{~B}$ & 108.00 \\
\hline $\mathrm{S} 1-\mathrm{C} 1-\mathrm{C} 6$ & $111.63(16)$ & $\mathrm{C} 8-\mathrm{C} 9-\mathrm{H} 9 \mathrm{~A}$ & 109.00 \\
\hline $\mathrm{S} 1-\mathrm{C} 1-\mathrm{C} 2$ & $128.03(16)$ & $\mathrm{C} 8-\mathrm{C} 9-\mathrm{H} 9 \mathrm{~B}$ & 109.00 \\
\hline $\mathrm{C} 1-\mathrm{C} 2-\mathrm{C} 3$ & $120.35(19)$ & $\mathrm{C} 10-\mathrm{C} 9-\mathrm{H} 9 \mathrm{~A}$ & 109.00 \\
\hline $\mathrm{C} 2-\mathrm{C} 3-\mathrm{C} 4$ & $118.6(2)$ & $\mathrm{C} 10-\mathrm{C} 9-\mathrm{H} 9 \mathrm{~B}$ & 109.00 \\
\hline $\mathrm{C} 11-\mathrm{C} 4-\mathrm{C} 3$ & $119.39(19)$ & $\mathrm{H} 9 \mathrm{~A}-\mathrm{C} 9-\mathrm{H} 9 \mathrm{~B}$ & 108.00 \\
\hline $\mathrm{C} 11-\mathrm{C} 4-\mathrm{C} 5$ & $118.43(17)$ & $\mathrm{C} 9-\mathrm{C} 10-\mathrm{H} 10$ & 107.00 \\
\hline $\mathrm{C} 3-\mathrm{C} 4-\mathrm{C} 5$ & $122.2(2)$ & $\mathrm{C} 11-\mathrm{C} 10-\mathrm{H} 10$ & 107.00 \\
\hline $\mathrm{C} 4-\mathrm{C} 5-\mathrm{C} 6$ & $118.9(2)$ & $\mathrm{C} 13-\mathrm{C} 10-\mathrm{H} 10$ & 107.00 \\
\hline $\mathrm{C} 1-\mathrm{C} 6-\mathrm{C} 5$ & $119.7(2)$ & $\mathrm{C} 10-\mathrm{C} 11-\mathrm{H} 11 \mathrm{~A}$ & 109.00 \\
\hline $\mathrm{N} 1-\mathrm{C} 6-\mathrm{C} 1$ & $114.0(2)$ & $\mathrm{C} 10-\mathrm{C} 11-\mathrm{H} 11 \mathrm{~B}$ & 109.00 \\
\hline $\mathrm{N} 1-\mathrm{C} 6-\mathrm{C} 5$ & $126.29(19)$ & $\mathrm{C} 12-\mathrm{C} 11-\mathrm{H} 11 \mathrm{~A}$ & 109.00 \\
\hline $\mathrm{S} 1-\mathrm{C} 7-\mathrm{N} 1$ & $103.38(15)$ & $\mathrm{C} 12-\mathrm{C} 11-\mathrm{H} 11 \mathrm{~B}$ & 109.00 \\
\hline $\mathrm{N} 1-\mathrm{C} 7-\mathrm{C} 8$ & $111.46(17)$ & $\mathrm{H} 11 \mathrm{~A}-\mathrm{C} 11-\mathrm{H} 11 \mathrm{~B}$ & 108.00 \\
\hline $\mathrm{S} 1-\mathrm{C} 7-\mathrm{C} 8$ & $110.32(15)$ & $\mathrm{C} 7-\mathrm{C} 12-\mathrm{H} 12 \mathrm{~A}$ & 109.00 \\
\hline $\mathrm{S} 1-\mathrm{C} 7-\mathrm{C} 12$ & $109.96(13)$ & $\mathrm{C} 7-\mathrm{C} 12-\mathrm{H} 12 \mathrm{~B}$ & 109.00 \\
\hline $\mathrm{C} 8-\mathrm{C} 7-\mathrm{C} 12$ & $109.82(19)$ & $\mathrm{C} 11-\mathrm{C} 12-\mathrm{H} 12 \mathrm{~A}$ & 109.00 \\
\hline $\mathrm{N} 1-\mathrm{C} 7-\mathrm{C} 12$ & $111.75(19)$ & $\mathrm{C} 11-\mathrm{C} 12-\mathrm{H} 12 \mathrm{~B}$ & 109.00 \\
\hline $\mathrm{C} 7-\mathrm{C} 8-\mathrm{C} 9$ & $113.43(16)$ & $\mathrm{H} 12 \mathrm{~A}-\mathrm{C} 12-\mathrm{H} 12 \mathrm{~B}$ & 108.00 \\
\hline $\mathrm{C} 8-\mathrm{C} 9-\mathrm{C} 10$ & $111.89(18)$ & $\mathrm{C} 13-\mathrm{C} 14-\mathrm{H} 14 \mathrm{~A}$ & 110.00 \\
\hline $\mathrm{C} 9-\mathrm{C} 10-\mathrm{C} 11$ & $107.78(18)$ & $\mathrm{C} 13-\mathrm{C} 14-\mathrm{H} 14 \mathrm{~B}$ & 109.00 \\
\hline $\mathrm{C} 9-\mathrm{C} 10-\mathrm{C} 13$ & $115.16(18)$ & $\mathrm{C} 13-\mathrm{C} 14-\mathrm{H} 14 \mathrm{C}$ & 109.00 \\
\hline $\mathrm{C} 11-\mathrm{C} 10-\mathrm{C} 13$ & $113.56(16)$ & $\mathrm{H} 14 \mathrm{~A}-\mathrm{C} 14-\mathrm{H} 14 \mathrm{~B}$ & 109.00 \\
\hline $\mathrm{C} 10-\mathrm{C} 11-\mathrm{C} 12$ & $112.56(16)$ & $\mathrm{H} 14 \mathrm{~A}-\mathrm{C} 14-\mathrm{H} 14 \mathrm{C}$ & 109.00 \\
\hline $\mathrm{C} 7-\mathrm{C} 12-\mathrm{C} 11$ & $112.28(18)$ & $\mathrm{H} 14 \mathrm{~B}-\mathrm{C} 14-\mathrm{H} 14 \mathrm{C}$ & 109.00 \\
\hline $\mathrm{C} 10-\mathrm{C} 13-\mathrm{C} 15$ & $109.36(19)$ & $\mathrm{C} 13-\mathrm{C} 15-\mathrm{H} 15 \mathrm{~A}$ & 110.00 \\
\hline $\mathrm{C} 10-\mathrm{C} 13-\mathrm{C} 16$ & $112.15(19)$ & $\mathrm{C} 13-\mathrm{C} 15-\mathrm{H} 15 \mathrm{~B}$ & 109.00 \\
\hline $\mathrm{C} 14-\mathrm{C} 13-\mathrm{C} 16$ & $108.1(2)$ & $\mathrm{C} 13-\mathrm{C} 15-\mathrm{H} 15 \mathrm{C}$ & 110.00 \\
\hline $\mathrm{C} 15-\mathrm{C} 13-\mathrm{C} 16$ & $108.70(18)$ & $\mathrm{H} 15 \mathrm{~A}-\mathrm{C} 15-\mathrm{H} 15 \mathrm{~B}$ & 110.00 \\
\hline $\mathrm{C} 14-\mathrm{C} 13-\mathrm{C} 15$ & $108.4(2)$ & $\mathrm{H} 15 \mathrm{~A}-\mathrm{C} 15-\mathrm{H} 15 \mathrm{C}$ & 109.00 \\
\hline $\mathrm{C} 10-\mathrm{C} 13-\mathrm{C} 14$ & $110.04(18)$ & $\mathrm{H} 15 \mathrm{~B}-\mathrm{C} 15-\mathrm{H} 15 \mathrm{C}$ & 109.00 \\
\hline $\mathrm{C} 1-\mathrm{C} 2-\mathrm{H} 2$ & 120.00 & $\mathrm{C} 13-\mathrm{C} 16-\mathrm{H} 16 \mathrm{~A}$ & 110.00 \\
\hline $\mathrm{C} 3-\mathrm{C} 2-\mathrm{H} 2$ & 120.00 & $\mathrm{C} 13-\mathrm{C} 16-\mathrm{H} 16 \mathrm{~B}$ & 109.00 \\
\hline $\mathrm{C} 2-\mathrm{C} 3-\mathrm{H} 3$ & 121.00 & $\mathrm{C} 13-\mathrm{C} 16-\mathrm{H} 16 \mathrm{C}$ & 109.00 \\
\hline $\mathrm{C} 4-\mathrm{C} 3-\mathrm{H} 3$ & 121.00 & $\mathrm{H} 16 \mathrm{~A}-\mathrm{C} 16-\mathrm{H} 16 \mathrm{~B}$ & 109.00 \\
\hline $\mathrm{C} 4-\mathrm{C} 5-\mathrm{H} 5$ & 121.00 & $\mathrm{H} 16 \mathrm{~A}-\mathrm{C} 16-\mathrm{H} 16 \mathrm{C}$ & 109.00 \\
\hline $\mathrm{C} 6-\mathrm{C} 5-\mathrm{H} 5$ & 121.00 & $\mathrm{H} 16 \mathrm{~B}-\mathrm{C} 16-\mathrm{H} 16 \mathrm{C}$ & 110.00 \\
\hline $\mathrm{C} 7-\mathrm{S} 1-\mathrm{C} 1-\mathrm{C} 2$ & $180.00(19)$ & $\mathrm{C} 4-\mathrm{C} 5-\mathrm{C} 6-\mathrm{N} 1$ & $178.9(2)$ \\
\hline $\mathrm{C} 7-\mathrm{S} 1-\mathrm{C} 1-\mathrm{C} 6$ & $-0.14(15)$ & $\mathrm{C} 4-\mathrm{C} 5-\mathrm{C} 6-\mathrm{C} 1$ & $-0.5(3)$ \\
\hline $\mathrm{C} 1-\mathrm{S} 1-\mathrm{C} 7-\mathrm{N} 1$ & $-1.11(14)$ & $\mathrm{S} 1-\mathrm{C} 7-\mathrm{C} 8-\mathrm{C} 9$ & $-68.5(2)$ \\
\hline $\mathrm{C} 1-\mathrm{S} 1-\mathrm{C} 7-\mathrm{C} 8$ & $-120.40(16)$ & $\mathrm{N} 1-\mathrm{C} 7-\mathrm{C} 8-\mathrm{C} 9$ & $177.21(19)$ \\
\hline $\mathrm{C} 1-\mathrm{S} 1-\mathrm{C} 7-\mathrm{C} 12$ & $118.33(16)$ & $\mathrm{C} 12-\mathrm{C} 7-\mathrm{C} 8-\mathrm{C} 9$ & $52.8(2)$ \\
\hline $\mathrm{C} 6-\mathrm{N} 1-\mathrm{C} 7-\mathrm{S} 1$ & $2.3(2)$ & $\mathrm{S} 1-\mathrm{C} 7-\mathrm{C} 12-\mathrm{C} 11$ & 69.25 (19) \\
\hline
\end{tabular}




$\begin{array}{llll}\mathrm{C} 6-\mathrm{N} 1-\mathrm{C} 7-\mathrm{C} 8 & 120.8(2) & \mathrm{N} 1-\mathrm{C} 7-\mathrm{C} 12-\mathrm{C} 11 & -176.55(16) \\ \mathrm{C} 6-\mathrm{N} 1-\mathrm{C} 7-\mathrm{C} 12 & -115.9(2) & \mathrm{C} 8-\mathrm{C} 7-\mathrm{C} 12-\mathrm{C} 11 & -52.3(2) \\ \mathrm{C} 7-\mathrm{N} 1-\mathrm{C} 6-\mathrm{C} 1 & -2.6(3) & \mathrm{C} 7-\mathrm{C} 8-\mathrm{C} 9-\mathrm{C} 10 & -56.6(2) \\ \mathrm{C} 7-\mathrm{N} 1-\mathrm{C} 6-\mathrm{C} 5 & 178.01(19) & \mathrm{C} 8-\mathrm{C} 9-\mathrm{C} 10-\mathrm{C} 11 & 56.2(2) \\ \mathrm{S} 1-\mathrm{C} 1-\mathrm{C} 2-\mathrm{C} 3 & 179.60(16) & \mathrm{C} 8-\mathrm{C} 9-\mathrm{C} 10-\mathrm{C} 13 & -175.93(15) \\ \mathrm{C} 6-\mathrm{C} 1-\mathrm{C} 2-\mathrm{C} 3 & -0.3(3) & \mathrm{C} 9-\mathrm{C} 10-\mathrm{C} 11-\mathrm{C} 12 & -56.7(2) \\ \mathrm{S} 1-\mathrm{C} 1-\mathrm{C} 6-\mathrm{N} 1 & 1.5(2) & \mathrm{C} 13-\mathrm{C} 10-\mathrm{C} 11-\mathrm{C} 12 & 174.45(17) \\ \mathrm{S} 1-\mathrm{C} 1-\mathrm{C} 6-\mathrm{C} 5 & -179.09(15) & \mathrm{C} 9-\mathrm{C} 10-\mathrm{C} 13-\mathrm{C} 14 & 46.7(2) \\ \mathrm{C} 2-\mathrm{C} 1-\mathrm{C} 6-\mathrm{N} 1 & -178.61(18) & \mathrm{C} 9-\mathrm{C} 10-\mathrm{C} 13-\mathrm{C} 15 & 165.71(17) \\ \mathrm{C} 2-\mathrm{C} 1-\mathrm{C} 6-\mathrm{C} 5 & 0.8(3) & \mathrm{C} 9-\mathrm{C} 10-\mathrm{C} 13-\mathrm{C} 16 & -73.6(2) \\ \mathrm{C} 1-\mathrm{C} 2-\mathrm{C} 3-\mathrm{C} 4 & -0.6(3) & \mathrm{C} 11-\mathrm{C} 10-\mathrm{C} 13-\mathrm{C} 14 & 171.7(2) \\ \mathrm{C} 2-\mathrm{C} 3-\mathrm{C} 4-\mathrm{C} 5 & 0.9(3) & \mathrm{C} 11-\mathrm{C} 10-\mathrm{C} 13-\mathrm{C} 15 & -69.4(2) \\ \mathrm{C} 2-\mathrm{C} 3-\mathrm{C} 4-\mathrm{C} 11 & -178.66(16) & \mathrm{C} 11-\mathrm{C} 10-\mathrm{C} 13-\mathrm{C} 16 & 51.3(2) \\ \mathrm{C} 11-\mathrm{C} 4-\mathrm{C} 5-\mathrm{C} 6 & 179.20(15) & \mathrm{C} 10-\mathrm{C} 11-\mathrm{C} 12-\mathrm{C} 7 & 56.6(2) \\ \mathrm{C} 3-\mathrm{C} 4-\mathrm{C} 5-\mathrm{C} 6 & -0.4(3) & & \end{array}$

Hydrogen-bond geometry $\left(A,{ }^{\circ}\right)$

$\mathrm{Cg} 1$ is the centroid of the $\mathrm{Cl} 1-\mathrm{C} 6$ benzene ring.

\begin{tabular}{lllll}
\hline$D-\mathrm{H} \cdots A$ & $D-\mathrm{H}$ & $\mathrm{H} \cdots A$ & $D \cdots A$ & $D-\mathrm{H}^{\cdots} A A$ \\
\hline $\mathrm{C} 8-\mathrm{H} 8 B^{\cdots} \cdots C g 1^{\mathrm{i}}$ & 0.97 & 2.84 & $3.796(2)$ & 169 \\
\hline
\end{tabular}

Symmetry code: (i) $-x, y+1 / 2,-z+1 / 2$. 